\title{
Canonical wave packets in quantum cosmology
}

\author{
S. S. Gousheh, H. R. Sepangi, P. Pedram* M. Mirzaei, \\ Department of Physics, Shahid Beheshti University, Evin, Tehran 19839, Iran
}

October 16, 2018

\begin{abstract}
We discuss the construction of wave packets resulting from the solutions of a class of Wheeler-DeWitt equations in Robertson-Walker type cosmologies, for arbitrary curvature. We show that there always exists a "canonical initial slope" for a given initial wave function, which optimizes some desirable properties of the resulting wave packet, most importantly good classical-quantum correspondence. This can be properly denoted as a canonical wave packet. We introduce a general method for finding these canonical initial slopes which is generalization of our earlier work.
\end{abstract}

PACS numbers: 04.20.Ex, 04.60.Kz, 04.25.-g

\section{Introduction}

The question of the construction and interpretation of wave packets in quantum cosmology and its connection with classical cosmology has been attracting much attention in recent years. Moreover, numerous studies have been done to obtain a quantum theory for gravity and to understand its connection with classical physics. The problem of the relation between quantum cosmology and classical physics is an important one that exists even in simple models [1]. Most authors consider semi-classical approximations to the Wheeler-DeWitt (WD) equation and refer to regions in configuration space where these solutions are oscillatory or exponentially decaying as representing classically allowed or forbidden regions, respectively. These regions are mainly determined by the initial conditions for the wave function. Two popular proposals for the initial conditions are the no boundary proposal [2] and the spontaneous nucleation from nothing [3]. These proposals have been attractive to many authors because they lead to some classes of classical solutions represented by certain trajectories which posses important features such as predicting an inflationary phase.

In quantum cosmology, in analogy with ordinary quantum mechanics, one is generally concerned with the construction of wave functions by the superposition of the 'energy eigenstates' which would peak around the

\footnotetext{
*Email: pedram@sbu.ac.ir
} 
classical trajectories in configuration space, whenever such classical-quantum correspondence is mandated by the nature of the problem. However, contrary to ordinary quantum mechanics, a parameter describing time is absent in quantum cosmology. Therefore, the initial conditions would have to be expressed in terms of an intrinsic time parameter, which in the case of the WD equation could be taken as the local scale factor for the three geometry [4. Also, since the sign of the kinetic term for the scale factor is negative, a formulation of the Cauchy problem for the WD equation is possible. The existence of such a sign is one of the exclusive features of gravity with many other interesting implications.

The construction of wave packets resulting from the solutions of the WD equation has been a feature common to most of the recent research work in quantum cosmology [5, 6, 7]. In particular, in reference [5] the construction of wave packets in a Friedmann universe is presented in detail and appropriate boundary conditions are motivated. Generally speaking one of the aims of these investigations has been to find wave packets whose probability distributions coincide with the classical paths obtained in classical cosmology. In these works, the authors usually consider model theories in which a self interacting scalar field is coupled to gravity in a Robertson-Walker type universe with zero $k$. The resulting WD equation is often in the form of an isotropic oscillator-ghost-oscillator and is separable in the configuration space variables. The general solution can thus be written as a sum over the product of simple harmonic oscillator wave functions with different frequencies. As usual, the coefficients in the sum and hence the exact form of the wave packets are determined by the initial conditions, which are usually specified by giving the wave function and its derivative at $R=0$, where $R$ is the scale factor. The coefficient are chosen such that the following desirable properties are obtained. Firstly, there should be a good classical-quantum correspondence, which means the wave packet should have compact support centered around the classical path, the crest of the wave packet should follow as closely as possible the classical path, and to each distinct classical path there should correspond a wave packet with the above properties. The first part of this condition implies that the initial wave function should consist of a few localized pieces. Secondly, one expects the square of the wave packet describing a physical system to posses a certain degree of smoothness.

At the classical level, if the initial position is known, the corresponding momentum can be calculated from the constraint $H=0$. However, in quantum cosmology the WD equation can be in general put in the form of 
a second order hyperbolic PDE. Therefore, complete description of the initial condition would in general entail the specification of both the initial wave function and its first derivative. However, it is in general an unresolved problem as to how, by knowing the initial classical values of the positions and momenta, one may embark on obtaining the corresponding quantum distributions. Generally speaking, there is no generic way of doing this. What is sometimes done is to consider a distribution which peaks around the classical values of the variables of interest, mostly and for simplicity taken as a Gaussian distribution, and simply let the resulting wave packet evolve and hope for a good classical quantum correspondence [8, 9]. Indeed, even if the wave packet does follow the classical path it might not be smooth. Smooth wave packets have been constructed in the past by adapting coherent states for the initial wave function and the undetermined coefficient related to the initial first derivative distribution are chosen to have the same functional form as those of the initial wave function [10. Here, we shall generalize the aforementioned prescription to be applicable to any reasonable potential in the same class of problems. Moreover, the prescription is general enough to be applicable to problems which are not exactly solvable. The situation is therefore becomes analogous to the classical case: Given an initial position distribution, there is a canonical initial momentum distribution which ensures good classicalquantum correspondence. This is in contrast to the usual practice where there is no uniform prescription for choosing initial momentum distribution in order to obtain a good classical quantum correspondence as possible is obtained. Our solution here is based on [10] where a certain prescription was offered for obtaining initial canonical slope.

The paper is organized as follows: In Sec. 2 we outline the main problem which is a case of RobertsonWalker type cosmology where the matter is taken to be a particular type of self-interacting scalar field. We derive the main equations both for the classical cosmology and the quantum one. In Sec. 3, we present a general prescription for finding a Canonical Initial Slope (CIS) for a given choice of suitable initial condition on the wave function, which produces the above desired properties of the wave packet. In this derivation we will come up with a decisive criteria for what initial wave functions are suitable for classical-quantum correspondence. This prescription is general enough to be applicable to both exactly solvable cases and the ones which are not. Therefore, we begin Sec. 3 with a description the Spectral Method (SM) [11] which is a robust numerical method. We then present the $k=0$ case which is exactly solvable and display the resulting wave packet 
obtained by CIS. Then we consider the case $k \neq 0$, for which neither the classical nor the quantum equations of motion are exactly solvable. In particular, in our model the classical equations of motion turn out to be a set of non-linear coupled ODEs with moving singularities. For the classical cases, we solve them by a numerical method that we introduced in an earlier work [12. In the quantum cosmology case we solve the problem using SM. For this case we display the resulting wave packets obtained by the CIS prescription. In Sec. 4 we draw some final conclusions.

\section{Robertson-Walker cosmology with a coupled scalar field}

In Robertson-Walker cosmology, one often considers the coupling of a scalar field to gravity. The resulting field equations include a 'zero energy constraint'. The WD equation in quantum cosmology is the result of the quantization consistent with this constraint. Now we discuss our model and derive the field equations in the classical and quantum domains.

Consider the Einstein's field equation coupled to a scalar field

$$
\begin{aligned}
R_{\mu \nu}-\frac{1}{2} g_{\mu \nu} R & =\kappa T_{\mu \nu}(\phi), \\
\Delta^{2} \phi-\frac{\partial U}{\partial \phi} & =0
\end{aligned}
$$

where $T_{\mu \nu}$ is the energy-momentum tensor coupling the scalar field $\phi$ to gravity and $U$ is a scalar potential for the scalar field $\phi$. Hereafter, we shall work in a system of units in which $\kappa=1$. We parameterize the metric as

$$
g=-d t^{2}+R^{2}(t) \frac{\sum d r^{i} d r^{i}}{\left(1+k r^{2} / 4\right)^{2}}
$$

where $R(t)$ is the usual scale factor and $k=+1,0,-1$ corresponds to a closed, flat or open universe, respectively. The field equations resulting from (11) and (2) with the metric given by (3) can be written as

$$
\begin{aligned}
3\left[\left(\frac{\dot{R}}{R}\right)^{2}+\frac{k}{R^{2}}\right] & =\frac{\dot{\phi}^{2}}{2}+U(\phi), \\
2\left(\frac{\ddot{R}}{R}\right)+\left(\frac{\dot{R}}{R}\right)^{2}+\frac{k}{R^{2}} & =-\frac{\dot{\phi}^{2}}{2}+U(\phi), \\
\ddot{\phi}+3 \frac{\dot{R}}{R} \dot{\phi}+\frac{\partial U}{\partial \phi} & =0 .
\end{aligned}
$$

Here a dot represents differentiation with respect to time. We require the potential $U(\phi)$ to have natural

characteristics for small $\phi$, so that we may identify the coefficient of $\frac{1}{2} \phi^{2}$ in its Taylor expansion as a positive 
mass squared $m^{2}$, and $U(0)$ as a cosmological constant $\Lambda$. An interesting choice of $U(\phi)$ with three free parameters is $6,13,10,14,15$,

$$
U(\phi)=\Lambda+\frac{m^{2}}{2 \alpha^{2}} \sinh ^{2}(\alpha \phi)+\frac{b}{2 \alpha^{2}} \sinh (2 \alpha \phi)
$$

In the above expression $\Lambda$ may be identified with a cosmological constant, $m^{2}=\partial^{2} U /\left.\partial \phi^{2}\right|_{\phi=0}$ is a mass squared parameter, $b$ is a coupling constant, and $\alpha^{2}=\frac{3}{8}$. However, one of our main reasons for choosing this potential is that its exact solutions exist for both the classical and quantum cosmology for $k=0$ [10] and we want to solve the problem for $k \neq 0$ and compare the solutions to the corresponding ones for $k=0$. To do this, we have general numerical methods that can solve both the classical [12] and quantum cosmological [11] cases. More importantly, having the exact solution for $k=0$ and approximate solution for $k \neq 0$ for the same potential simultaneously, we can formulate a general procedure for finding the CIS and test it. Our claim would then be the resulting general formulation works for any potential in the same category. This gives us motivation to solve this problem for $k \neq 0$ for the case of quantum cosmology. The Lagrangian giving the above equations of motion can be written as

$$
L=-3 R \dot{R}^{2}+3 k R+R^{3}\left[\dot{\phi}^{2} / 2-U(\phi)\right]
$$

This Lagrangian can be cast into a simple form by using the transformations $X=R^{3 / 2} \cosh (\alpha \phi)$ and $Y=$ $R^{3 / 2} \sinh (\alpha \phi)$, which transform the term $R^{3} U(\phi)$ into a quadratic form. Upon using a second transformation to eliminate the coupling term in the quadratic form, we arrive at new variables $u$ and $v$, which are linear combinations of $X$ and $Y$,

$$
\left(\begin{array}{l}
u \\
v
\end{array}\right)=\left(\begin{array}{rr}
\cosh (\theta) & -\sinh (\theta) \\
-\sinh (\theta) & \cosh (\theta)
\end{array}\right)\left(\begin{array}{l}
X \\
Y
\end{array}\right)
$$

where,

$$
\theta=\frac{1}{2} \tanh ^{-1}\left(\frac{-2 b}{m^{2}}\right)
$$

In the terms of the new variables, the Lagrangian takes on the following simple form,

$$
L(u, v)=\frac{4}{3}\left[\left(\dot{u}^{2}-\omega_{1}^{2} u^{2}\right)-\left(\dot{v}^{2}-\omega_{2}^{2} v^{2}\right)-\frac{9}{4} k\left(u^{2}-v^{2}\right)^{1 / 3}\right]
$$


where $\omega_{1,2}^{2}=-3 \lambda / 4+m^{2} / 2 \mp \sqrt{m^{4}-4 b^{2}} / 2$. The resulting classical field equations are,

$$
\begin{gathered}
\ddot{u}+\omega_{1}^{2} u+\frac{3 k}{2} \frac{u}{\left(u^{2}-v^{2}\right)^{2 / 3}}=0, \\
\ddot{v}+\omega_{2}^{2} v+\frac{3 k}{2} \frac{v}{\left(u^{2}-v^{2}\right)^{2 / 3}}=0, \\
\dot{u}^{2}+\omega_{1}^{2} u^{2}-\dot{v}^{2}-\omega_{2}^{2} v^{2}+\frac{9}{4} k\left(u^{2}-v^{2}\right)^{1 / 3}=0 .
\end{gathered}
$$

Equations (12) and (13) are the dynamical equations and (14) is the zero energy constraint. The non-linearity and the moving singular behavior of these equations are now apparent.

The corresponding quantum cosmology is described by the Wheeler-DeWitt (WD) equation written as,

$$
H \psi(u, v)=\left\{-\frac{\partial^{2}}{\partial u^{2}}+\frac{\partial^{2}}{\partial v^{2}}+\omega_{1}^{2} u^{2}-\omega_{2}^{2} v^{2}+\frac{9}{4} k\left(u^{2}-v^{2}\right)^{1 / 3}\right\} \psi(u, v)=0
$$

which arises from the zero energy condition Eq. (14).

\section{Solutions for the classical and quantum cosmology cases}

In this section we shall present a general prescription for finding CIS. The cases that we shall study will include some cases which are exactly solvable $(k=0)$ and some which are not $(k \neq 0)$. For the latter we have to resort in part to some numerical methods. Therefore, we start this section by a discussion of the numerical method that we shall use, so that later on we can concentrate on the physics of the problem with no interruption. The general PDE that we want to solve is

$$
\left\{-\frac{\partial^{2}}{\partial u^{2}}+\frac{\partial^{2}}{\partial v^{2}}+\hat{f}^{\prime}(u, v)\right\} \psi(u, v)=0,
$$

where $\hat{f}^{\prime}(u, v)$ is an arbitrary function. This equation has the general form of a hyperbolic PDE equation and is encountered in many branches of science and in particular physics. It is notable that such equations may represent a wave-like equation whose solution may rapidly oscillate. In such cases, the usual spatial integration routines such as Finite Difference Methods fail to produce a reasonable solution. It is therefore of prime importance to introduce a method of solution which is efficient, accurate, predictable and easily implemented, given any reasonable boundary conditions $[11$. 
Equation (15) in general is not separable except when $k=0$ [10], and we have to resort to a numerical method to solve this hyperbolic PDE. Among the various numerical techniques, we choose SM which has the following advantages: It is very simple, fast, accurate, robust and stable. Spectral Method (SM) [16, 17, 18, consists of first choosing a complete orthonormal set of eigenstates of a, preferably relevant, hermitian operator to be used as a suitable basis for our solution. For this numerical method we obviously cannot choose the whole set of the complete basis, as these are usually infinite. Therefore we make the approximation of representing the solution by only a finite superposition of the basis functions. By substituting this approximate solution into the differential equation, a matrix equation is obtained. The expansion coefficients of these approximate solutions could be determined by eigenfunctions of this matrix. In this method, the accuracy of the solution is increased by choosing a larger set of basis functions. Having resorted to a numerical method, it is worth setting up a more general problem defined by the following WD equation,

$$
H \psi(u, v)=\left\{-\frac{\partial^{2}}{\partial u^{2}}+\frac{\partial^{2}}{\partial v^{2}}+\omega_{1}^{2} u^{2}-\omega_{2}^{2} v^{2}+\hat{f}(u, v)\right\} \psi(u, v)=0 .
$$

As mentioned before, any complete orthonormal set can be used. In this section we use the Fourier series basis by restricting the configuration space to a finite square region of sides $2 L$. This means that we can expand the solution as,

$$
\psi(u, v)=\sum_{i, j=1}^{2} \sum_{m, n} A_{m, n, i, j} g_{i}\left(\frac{m \pi u}{L}\right) g_{j}\left(\frac{n \pi v}{L}\right),
$$

where,

$$
\left\{\begin{array}{l}
g_{1}\left(\frac{m \pi u}{L}\right)=\sqrt{\frac{2}{R_{m} L}} \sin \left(\frac{m \pi u}{L}\right), \\
g_{2}\left(\frac{m \pi u}{L}\right)=\sqrt{\frac{2}{R_{m} L}} \cos \left(\frac{m \pi u}{L}\right) .
\end{array} \quad \text { and } R_{m}= \begin{cases}1, & m \neq 0 \\
2, & m=0\end{cases}\right.
$$

By referring to the WD equation (16), we realize that in the Fourier basis it is appropriate to introduce $\hat{f}^{\prime}$ as,

$$
\hat{f}^{\prime}(u, v)=\hat{f}(u, v)+\omega_{1}^{2} u^{2}-\omega_{2}^{2} v^{2} .
$$

We can make the following expansion,

$$
\hat{f}^{\prime}(u, v) \psi(u, v)=\sum_{i, j} \sum_{m, n} B_{m, n, i, j}^{\prime} g_{i}\left(\frac{m \pi u}{L}\right) g_{j}\left(\frac{n \pi v}{L}\right)
$$

where $B_{m, n, i, j}^{\prime}$ are coefficients that can be determined once $\hat{f}^{\prime}(u, v)$ is specified. By substituting (17/20) in (16), 
and using independence of $g_{i}\left(\frac{m \pi u}{L}\right) \mathrm{s}$ and $g_{j}\left(\frac{n \pi v}{L}\right) \mathrm{s}$ we obtain,

$$
\left[\left(\frac{m \pi}{L}\right)^{2}-\left(\frac{n \pi}{L}\right)^{2}\right] A_{m, n, i, j}+B_{m, n, i, j}^{\prime}=0
$$

where,

$$
\begin{aligned}
B_{m, n, i, j}^{\prime} & =\sum_{m^{\prime}, n^{\prime}, i^{\prime}, j^{\prime}}\left[\iint_{-L}^{L} g_{i}\left(\frac{m \pi u}{L}\right) g_{j}\left(\frac{n \pi v}{L}\right) \hat{f}^{\prime}(u, v) g_{i^{\prime}}\left(\frac{m^{\prime} \pi u}{L}\right) g_{j^{\prime}}\left(\frac{n^{\prime} \pi v}{L}\right) d u d v\right] A_{m^{\prime}, n^{\prime}, i^{\prime}, j^{\prime}} \\
& =\sum_{m^{\prime}, n^{\prime}, i^{\prime}, j^{\prime}} C_{m, n, i, j, m^{\prime}, n^{\prime}, i^{\prime}, j^{\prime}}^{\prime} A_{m^{\prime}, n^{\prime}, i^{\prime}, j^{\prime}}
\end{aligned}
$$

Therefore we can rewrite (21) as

$$
\left[\left(\frac{m \pi}{L}\right)^{2}-\left(\frac{n \pi}{L}\right)^{2}\right] A_{m, n, i, j}+\sum_{m^{\prime}, n^{\prime}, i^{\prime}, j^{\prime}} C_{m, n, i, j, m^{\prime}, n^{\prime}, i^{\prime}, j^{\prime}}^{\prime} A_{m^{\prime}, n^{\prime}, i^{\prime}, j^{\prime}}=0
$$

In this case, we select $4 N^{2}$ basis functions, that is $m$ and $n$ run from 1 to $N$. It is obvious that the presence of the operator $\hat{f}^{\prime}(u, v)$ leads to nonzero coefficients $C_{m, n, i, j, m^{\prime}, n^{\prime}, i^{\prime}, j^{\prime}}^{\prime}$ in (23), which in principle could couple all of the matrix elements of $A$. Then we replace the square matrix $A$ with a column vector $A^{\prime}$ with $(2 N)^{2}$ elements, so that any element of $A$ corresponds to one element of $A^{\prime}$. This transforms (23) to

$$
D A^{\prime}=0
$$

Matrix $D$ is a square matrix now with $(2 N)^{2} \times(2 N)^{2}$ elements which can be easily obtained from (23). Equation (24) can be looked upon as an eigenvalue equation, i.e. $D A_{a}^{\prime}=a A_{a}^{\prime}$ and the matrix $D$ has $(2 N)^{2}$ eigenvectors. However, for constructing the acceptable wavefunctions, i.e. the ones satisfying the WD equation (16), we only require eigenvectors which span the null space of the matrix $D$. That is, due to (23) we will have exactly $2 N$ null eigenvectors which will be linear combination of our original eigenfunctions introduced in (17). After finding the $2 N$ eigenvectors of $D$ with zero eigenvalue, i.e. $A^{\prime k}(k=1,2,3, \ldots, 2 N)$, we can find the corresponding elements of matrix $A, A_{m, n, i, j}^{k}$. Therefore, the wavefunction can be expanded as

$$
\psi(u, v)=\sum_{k} \lambda^{k} \psi^{k}(u, v)=\sum_{k} \lambda^{k} \sum_{m, n, i, j} A_{m, n, i, j}^{k} g_{i}\left(\frac{m \pi u}{L}\right) g_{j}\left(\frac{n \pi v}{L}\right) .
$$

Here $\lambda^{k} \mathrm{~s}$ in (25) are arbitrary complex constants to be determined by the initial conditions.

We are free to adjust two parameters: $2 N$, the number of basis elements used and the length of the spatial region, $2 L$. This length should be preferably larger than spatial spreading of all the sought after wave functions. 
However, if $2 L$ is chosen to be too large we loose overall accuracy. Therefore it is important to note that for each $N, L$ can be properly adjusted [16].

The most important physical aspects of this problem that we want to address is on the question of the existence of a CIS. In our previous investigation of the same problem for $k=0$ [10] we discovered that a "canonical" choice for the slope exists whose use produces wave packets with all the desired properties stated in the introduction. Here we have come up with a more general and systematic method for obtaining this slope. The results are based on the same general prescription as before and obviously give identical results for comparable cases. This slope turns out to depend on the nature of the hamiltonian and the initial wave function. Since the WD equation is a hyperbolic PDE, for any reasonable choice of initial wave packet, there will remain certain undetermined coefficients which will be pending the specification of the initial first derivative. Our prescription is simply to choose the undetermined coefficient to have the same functional form as the determined ones. We shall clarify this prescription further in some concrete examples. If the problem is exactly solvable this slope can be easily obtained by the method described in Ref. [10. If it is not, we can use the following prescription: We use the minimum approximation necessary to make the general PDE (i.e. (16)) separable near $v=0$ and solve the resulting equations. It is obvious that the presence of the odd terms of $v$ dose not have any effect on the form of the initial wave function but they are responsible for the slope of the wave function at $v=0$, and vice versa for the even terms. We can approximate (15) near the $v=0$, so up to the first order in $v$ we have,

$$
\left\{-\frac{\partial^{2}}{\partial u^{2}}+\frac{\partial^{2}}{\partial v^{2}}+\omega_{1}^{2} u^{2}+\frac{9}{4} k\left(u^{2}\right)^{1 / 3}\right\} \psi(u, v)=0
$$

This PDE is separable in $u$ and $v$ variables, so we can write,

$$
\psi(u, v)=\varphi(u) \chi(v)
$$

By using this definition in (26), two ODEs can be derived,

$$
\begin{aligned}
\frac{d^{2} \chi_{n}(v)}{d v^{2}}+E_{n} \chi_{n}(v) & =0 \\
-\frac{d^{2} \varphi_{n}(u)}{d u^{2}}+\left(\omega_{1}^{2} u^{2}+\frac{9}{4} k u^{2 / 3}\right) \varphi_{n}(u) & =E_{n} \varphi_{n}(u),
\end{aligned}
$$

where $E_{n} \mathrm{~s}$ are separation constants. These equations are Schrödinger-like equations with $E_{n} \mathrm{~s}$ as their 'energy' 
levels. Equation (28) is exactly solvable with plane wave solutions or,

$$
\chi_{n}(v)=\alpha_{n} \cos \left(\sqrt{E_{n}} v\right)+i \beta_{n} \sin \left(\sqrt{E_{n}} v\right)
$$

where $\alpha_{n}$ and $\beta_{n}$ are arbitrary complex numbers. Equation (29) does not seem to be exactly solvable and we resort to a numerical technique. As mentioned before, SM can be used to find the bound state energy levels $\left(E_{n}\right)$ and the corresponding wave functions $\left(\varphi_{n}(u)\right)$ with high accuracy. The general solution to the (26) can be written as,

$$
\psi(u, v)=\sum_{n=\text { even }}\left(A_{n} \cos \left(\sqrt{E_{n}} v\right)+i B_{n} \sin \left(\sqrt{E_{n}} v\right)\right) \varphi_{n}(u)+\sum_{n=\text { odd }}\left(C_{n} \cos \left(\sqrt{E_{n}} v\right)+i D_{n} \sin \left(\sqrt{E_{n}} v\right)\right) \varphi_{n}(u)
$$

The separation of this solution to even and odd terms, though in principle unnecessary, is crucial for our prescription for the CIS. As stated before, this solution is valid only for small $v$. The general initial conditions can now be written as,

$$
\begin{array}{r}
\psi(u, 0)=\sum_{\text {even }} A_{n} \varphi_{n}(u)+\sum_{\text {odd }} C_{n} \varphi_{n}(u) \\
\psi^{\prime}(u, 0)=i \sum_{\text {even }} B_{n} \sqrt{E_{n}} \varphi_{n}(u)+i \sum_{\text {odd }} D_{n} \sqrt{E_{n}} \varphi_{n}(u),
\end{array}
$$

where prime denotes the derivative with respect to $v$. Obviously a complete description of the problem would include the specification of both these quantities. However, given only the initial condition on the wave function, we claim there is only a CIS which produces a canonical wave packet with all the aforementioned desired properties. We can qualitatively describe our prescription for this case as setting the functional form of the odd undetermined coefficients to be the same as the even determined ones and vice versa. This means that the coefficients that determine CIS i.e. $B_{n}$ for $n$ even and $D_{n}$ for $n$ odd, are chosen by the following,

$$
B_{n}=C_{n} \quad \text { for } n \text { even } \quad D_{n}=A_{n} \text { for } n \text { odd }
$$

To be more specific, although $C_{n}\left(A_{n}\right)$ is defined only for $n$ odd (even), we can extend its definition to $n$ even (odd) by choosing the same functional form.

Now we want to discuss the general settings for quantum-classical correspondence. As mentioned before in the classical cosmology case, the initial condition is given by specifying a few isolated initial points in the configuration space and uniquely determined velocities associated with each one through the constraint $H=0$. 
In order to established a good quantum-classical correspondence, the first thing we should do is to specify an initial wave function with isolated peaks centered around the classical ones. Then we can use the CIS prescription to obtain the initial first derivative distribution. One of the main outcomes of our prescription is that the expectation value of initial momentum computed using our prescription in the quantum cosmology case, denoted by $\left[\left\langle p_{v}\right\rangle\right]_{v=0}$, is approximately the same as the corresponding classical quantity $[\dot{v}(t)]_{t=0}$ (in our notation) for each isolated classical point. That is $\left[\left\langle p_{v}\right\rangle\right]_{v=0} \rightarrow[\dot{v}(t)]_{t=0}$ where,

$$
\left[\left\langle p_{v}\right\rangle\right]_{v=0}=\int\left[\psi(u, v)^{*}\left(-i \frac{\partial}{\partial v}\right) \psi(u, v)\right]_{v=0}
$$

Obviously the domain of the above integral should extend only over the appropriate part of the initial wave function, which corresponds to the classical point in question. This relationship approaches an equality for initial states for which classical description is appropriate. We shall shortly make this statement more explicit for the particular problem under investigation in this paper. Our prescription for the CIS is general enough to be applicable to any initial wave function.

In the case $k=0$ the problem is exactly solvable [10] and has a closed form solution in the Simple Harmonic Oscillator (SHO) basis. In references [5, 10, the authors considered the following initial condition for the wave function,

$$
\psi(u, 0)=\frac{1}{2 \pi^{1 / 4}}\left(e^{-(u-\chi)^{2} / 2}+e^{-(u+\chi)^{2} / 2}\right)
$$

which consists of two symmetric coherent states of a one dimensional SHO. Note that, for the given choice of the initial wave function we can not expect a good classical-quantum correspondence for $\chi<3$, since there would be a significant overlap between the two pieces, which could cause quantum interference. With this choice for the initial wave function one might expect the classical-quantum correspondence to be manifest for $\chi>3$. However, as we shall see, this crucially depends on the choice of the initial slope. Obviously, this choice for $\psi(u, 0)$ is not unique and is chosen so that the resulting wave packet would have compact support. In the case $k=0$, (15) is exactly separable without any approximation and yields exactly the same Schrödinger-like equation, i.e. SHO, as the one obtained from (29) for both $u$ and $v$ variables with frequencies $\omega_{1}$ and $\omega_{2}$ respectively. The solutions to these equation are well known, i.e. for $u$ is

$$
\varphi_{n}^{0}(u)=\left(\frac{\omega_{1}}{\pi}\right)^{1 / 4} \frac{H_{n}\left(\sqrt{\omega_{1}} u\right)}{\sqrt{2^{n} n !}} e^{-\omega_{1} u^{2} / 2}, \quad E_{n}^{0}=(2 n+1) \omega_{1},
$$


where $H_{n} \mathrm{~s}$ are the Hermite polynomials and superscript zero indicates $k=0$. Then the complete eigenstates will be products of eigenstates for $u$ and $v$, with constraint that they should have the same energy. In this case the initial wave function is chosen to be even and taking into account our prescription for the CIS (34), (32/33) can be rewritten as,

$$
\begin{aligned}
\psi(u, 0) & =\sum_{\text {even }} c_{n} \varphi_{n}^{0}(u) \\
\psi^{\prime}(u, 0) & =i \sum_{\text {odd }} c_{n} \sqrt{(2 n+1) \omega_{1}} \varphi_{n}^{0}(u),
\end{aligned}
$$

where the choice,

$$
c_{n}=e^{-\frac{1}{4}|\chi|^{2}} \frac{\chi^{n}}{\sqrt{2^{n} n !}},
$$

produces our aforementioned wave function (36) and $\chi$ is an arbitrary complex number. The CIS introduced in Ref. [10], based on the same prescription as introduced here but for the exactly solvable case $k=0$, is,

$$
\psi^{\prime}(u, 0)=\sum_{n_{\text {odd }}} \frac{c_{n} \varphi_{n}^{0}(u) H_{n}^{\prime}(0)}{\frac{(-1)^{(n / 2)} n !}{(n / 2) !}}
$$

where prime denotes differentiation with respect to $v$. Numerical comparison between (39) and (41) shows that their relative difference is $\mathcal{O}\left(10^{-4}\right)$. Their difference is due to the fact that (41) is exact and (39) is approximate. We can now easily construct the coherent wave packets using (38) and (39) or (41). For illustrative purposes we choose the simplest case possible which is when $\omega_{1}=\omega_{2}$. The result is shown in the left part of Fig. 1. As can be seen from this figure the parameters of the problem are chosen such that the initial state consists of two well separated peaks and this class of problems are the ones which are also amenable to a classical description. We will make this statement more quantitative at the end of this section. We should mention that there are a variety of different cases illustrated in Ref. [10] for the same exactly solvable case of $k=0$, including $\omega_{1} \neq \omega_{2}$ obviously using (41).

The classical paths corresponding to these solutions can be easily obtained from (1213). The corresponding initial conditions for the classical case is the following:

$$
u(0)=-\chi, \quad v(0)=0, \quad \dot{u}(0)=0, \quad \dot{v}(0)=\dot{v}_{0},
$$

and the parameters $\chi$ and $\dot{v}_{0}$ are adjusted so that the zero energy condition (14) is satisfied. With this choice of initial conditions the exact classical paths would be, in the case $k=0$, Lissajous figures in general, which 
are circles with radii $\chi$ when $\omega_{1}=\omega_{2} \equiv \omega$. Using (35) we can compute the average initial momentum in the quantum case for the left peak, where the corresponding classical values are $u(0)=-\chi, v(0)=0, \dot{u}(0)=0$ and $\dot{v}=+\omega \chi$. It is important to note that the domain of integration in equation (35) in this case should be from $-\infty$ to zero, as explained before. We obtain the following two expressions first using the exact value for CIS (41), and second the approximate CIS (39),

$$
\begin{aligned}
{\left[\left\langle p_{v}\right\rangle\right]_{v=0}^{\text {exact }} } & =4 \sum_{m_{\text {odd }}} \sum_{n_{\text {even }}} \frac{c_{n} c_{m}}{\sqrt{2^{n} n ! \sqrt{\pi}} \sqrt{2^{m} m ! \sqrt{\pi}}}\left[\frac{(m / 2) ! \sqrt{\omega} H_{m}^{\prime}(0)}{(-1)^{((m+1) / 2)} m !}\right] n ! m ! \sum_{k=0}^{\operatorname{Min}\{m, n\}} \frac{2^{k} H(0)_{m+n-2 k-1}(43)}{k !(n-k) !(m-k) !} \\
{\left[\left\langle p_{v}\right\rangle\right]_{v=0}^{\text {approx. }} } & =4 \sum_{m_{\text {odd }}} \sum_{n_{\text {even }}} \frac{c_{n} c_{m}}{\sqrt{2^{n} n ! \sqrt{\pi}} \sqrt{2^{m} m ! \sqrt{\pi}}}[i \sqrt{(2 m+1) \omega}] n ! m ! \sum_{k=0}^{\operatorname{Min}\{m, n\}} \frac{2^{k} H(0)_{m+n-2 k-1}}{k !(n-k) !(m-k) !} \quad \text { (44) }
\end{aligned}
$$

Having precisely set the initial conditions for both the classical and quantum cosmology cases, we can now superimpose the results for the case $k=0$, as illustrated in the right part of the Fig. 1. As can be seen in the figure, and also for all the cases presented in [10], the classical-quantum correspondence is manifest. In Fig. 2 we show $\dot{v}(0)$ versus $\chi$ for the three cases: classical, exact CIS, and approximate CIS. As can be seen from this figure the classical-quantum correspondence is meaningful only for $\chi>3$, that is where there is no significant overlap between the two pieces of $\psi(u, 0)$. This correspondence becomes better as $\chi$ increases. That is $\left[\left\langle p_{v}\right\rangle\right]_{v=0}=[\dot{v}(t)]_{t=0}=\dot{v}_{0}$ for large $\chi$. We can generically expect loss of classical-quantum correspondence when there is significant quantum interference between different segments of the wave packet in the configuration space, when each segment is supposed to correspond to a distinct classical configuration. We would like to emphasize that the choice of CIS is crucial for establishing a good correlation. For example in Ref. [5] the initial slope in the quantum case is chosen to be zero, implying zero initial average momentum (see (35)), however the corresponding classical quantity $\dot{v}(0)= \pm \omega \chi \neq 0$ and the classical path would again be a circle. To be more precise, the WD equation emanates from the zero energy condition and their choice of initial conditions violates this constraint. Therefore it would be impossible to establish a classical-quantum correspondence in this case. It is interesting to note that their resulting wave packet $\left(|\psi(u, v)|^{2}\right)$ is wildly oscillating.

For the case $k \neq 0$, the problem is not exactly solvable in quantum cosmology and we will use the SM to get an approximate solution. We have to mention that the corresponding equations for classical cosmology, (12)-(14), are a set of nonlinear, coupled ODEs with moving singularities which are not exactly solvable either. 
However, a general method for solving them has been presented in [12, and this is the method we shall use. Also a detailed explanation of the physical setting of the problem in the classical domain has been presented in 19 . For ease of comparison, we choose the same illustrative problem with $\omega_{1}=\omega_{2}=\omega$ and the same coefficients for the initial wave functions as the case $k=0$ (i.e. (38|400). However, note that since the eigenstates $\phi_{n}^{ \pm 1}(u)$ and $\phi_{n}^{0}(u)$ (both obtainable from (29)) are slightly different, the resulting initial wave functions for $k= \pm 1$ would be slightly different from (36). From (29/33) it is apparent that the CISs of the wave functions are different in all cases $(k=0,1,-1)$, due to the differences both in $\phi_{n}(u) \mathrm{s}$ and $E_{n} \mathrm{~s}$. We have computed both the classical and quantum solutions for the $k=+1,-1$ cases and the results are shown in Figs. 3 and 4 respectively. Note that the wave packets are very smooth and we have good classical-quantum correspondence for both cases. Moreover, we have computed the graphs of $\dot{v}(0)$ versus $\chi$ for $k= \pm 1$ and have found the classical-quantum correspondence to be as good as that exhibited in Fig. 2 for $k=0$. In order to highlight the differences between the classical paths (and obviously the wave packets) we exhibit $r=\sqrt{u^{2}+v^{2}}$ versus $\theta=\arctan (v / u$ ) in Fig. 5 for the three cases.

\section{Conclusions}

We have described a Robertson-Walker type cosmology leading to classical dynamical equations given by (12. 14), and the corresponding WD equation represented by (15). All these equations are exactly solvable for the particular potential chosen when $k=0$ [10, and do not seem to be so when $k \neq 0$. In the latter cases we have solved these equations numerically: the classical cosmology equations by the numerical method introduced in [12, and the quantum cosmology case by an implementation of the SM [11. Most importantly, we have introduced a general method for finding the canonical initial slopes for any initial wave function and any value of $k$, whose use produces wave packets which can be named canonical wave packets. The latter have the following desirable properties: The correlation part of classical-quantum correspondence is acceptable, as outlined in detail in the Introduction. Moreover, the resulting wave packets are very smooth and this is usually considered to be a desirable property. Our investigation has also revealed that the classical-quantum correspondence is possible only when there is no overlap between the lumps of a suitably chosen initial wave function. Using the CIS, the average initial momenta become very close to their corresponding classical values in the limit where 
the overlap between the separate parts of the initial wave function goes to zero. It is important to note that our generalized method is also applicable to cases which are not exactly solvable.

\section{Acknowledgement}

This research has been supported by the office of research of Shahid Beheshti University under Grant No. $500 / 3787$.

\section{References}

[1] T. P. Singh and T. Padmanabhan, Ann. Phys. 196 (1989) 269.

[2] J. B. Hartle and S. W. Hawking, Phys. Rev. D 28 (1983) 2960;

S. W. Hawking, Nucl. Phys. B 239 (1984) 257.

[3] A. Vilenkin, Phys. Lett. B 117 (1982) 25; Phys. Rev. D 30 (1984) 549.

[4] B. S. DeWitt, Phys. Rev. 160 (1967) 1113.

[5] C. Kiefer, Nucl. Phys. B 341 (1990) 273;

C. Kiefer, Phys. Rev. D 38 (1988) 1761;

C. Kiefer, Phys. Lett. B 225 (1989) 227.

[6] T. Dereli, M. Onder and R. W. Tucker, Class. Quantum Grav. 10 (1993) 1425.

[7] F. Darabi and H. R. Sepangi, Class. Quantum Grav. 16 (1999) 1565.

[8] M. P. Dąbrowski, C. Kiefer, and B. Sandhöfer, Phys. Rev. D. 74, 044022 (2006).

[9] A. Ashtekar, T. Pawlowski, P. Singh, and K. Vandersloot, Phys. Rev. D. 75, 024035 (2007).

[10] S. S. Gousheh and H. R. Sepangi, Phys. Lett. A 272 (2000) 304-312.

[11] P. Pedram, M. Mirzaei and S. S. Gousheh, Computer Physics Communications, 176 (2007) 581.

[12] S. S. Gousheh, H. R. Sepangi and K. Ghafoori-Tabrizi, Computer Physics Communications 149 (2003) $135-141$. 
[13] F. Darabi, Phys. Lett. A 259, (1999) 97.

[14] F. Darabi, A. Rastkar, Gen. Rel. Grav. 38 (2006) 1355-1366.

[15] S. Jalalzadeh, F. Ahmadi, H. R. Sepangi, JHEP 0308 (2003) 012.

[16] J. P. Boyd, Chebyshev \& Fourier Spectral Methods, Springer-Verlag, BerlinHeidelberg, (1989).

[17] D. Gottleib and S. Ortega, Numerical analysis of spectral methods: theory and applications, SIAM, Philadelphia (1977).

[18] C. Canuto, M. Y. Hussaini, A. Quateroni and T. Zang, Spectral Methods in Fluid Dynamics Springer, Berlin (1988).

[19] K. Ghafoori-Tabrizi, S. S. Gousheh and H. R. Sepangi, Int. J. Mod. Phys. A 15 (2000) 1521. 

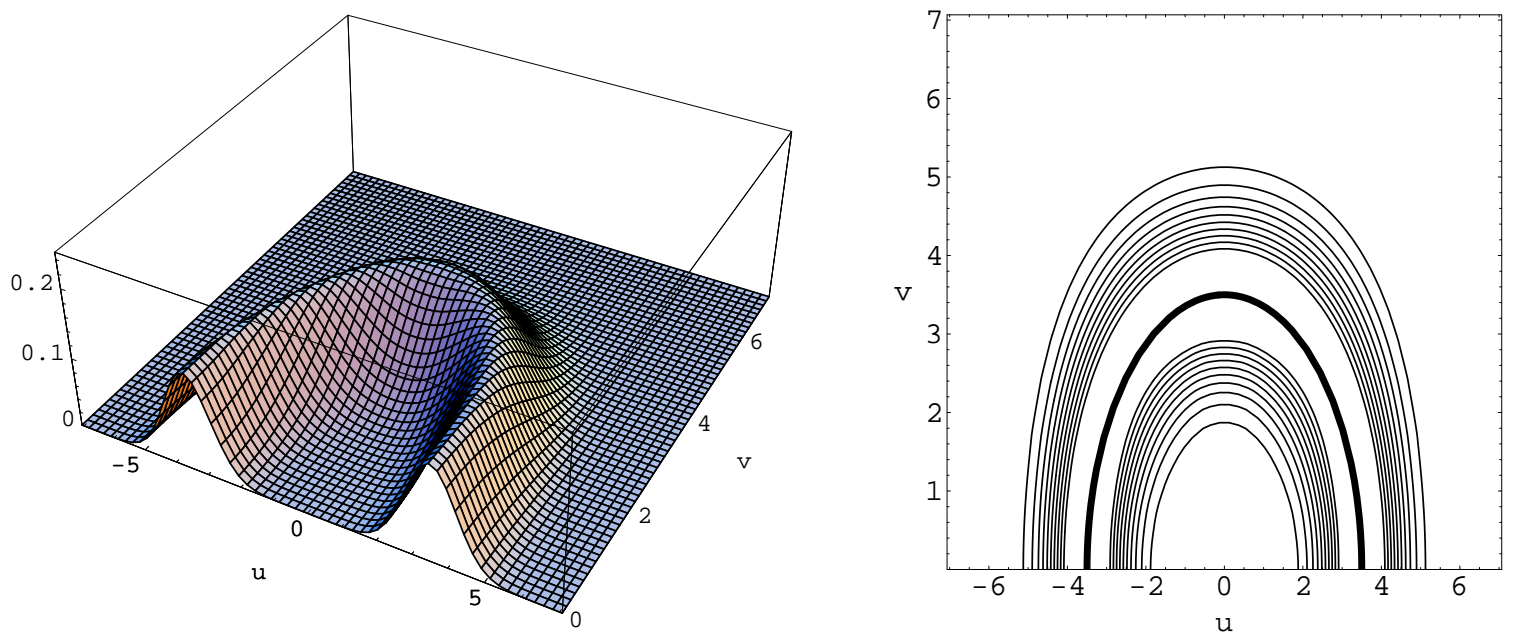

Figure 1: $k=0$ case: Left, the square of the wave packet $|\psi(u, v)|^{2}$ for $\chi=3.5$ and $N=15$. Right, the contour plot of the same figure with the classical path superimposed as the thick solid line.

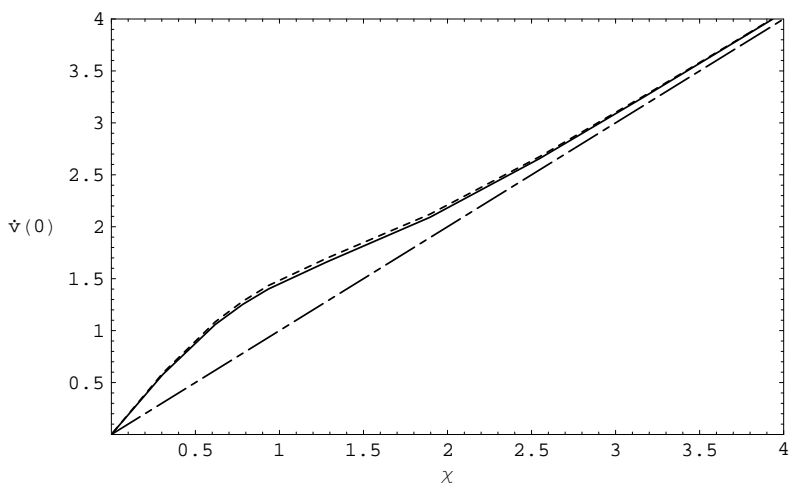

Figure 2: $\dot{v}(0)$ versus $\chi$ for the three cases: classical (dashed-dot), exact CIS (dashed), and approximate CIS (solid). On this scale the latter two approximately coincide for $\chi>4$. Their maximum difference is of order $10^{-2}$. These two CISs asymptotically approach the classical value for $\chi>8$.) 

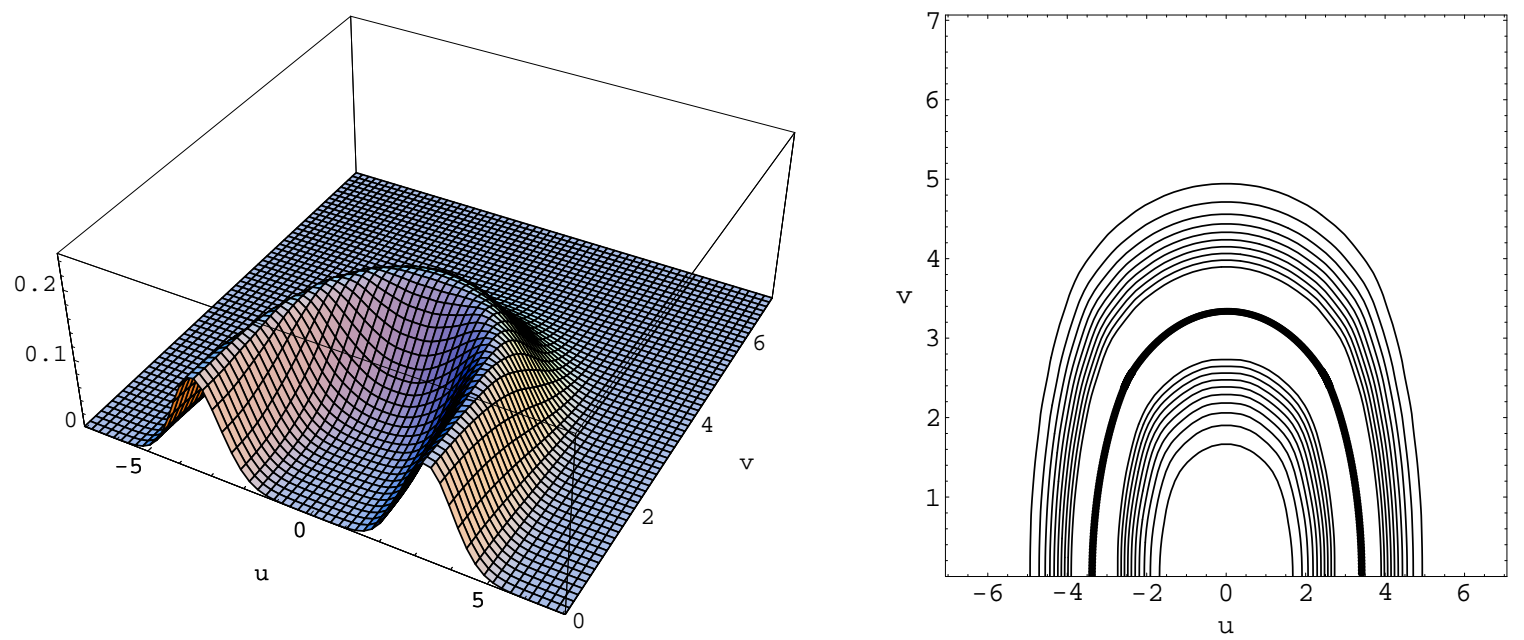

Figure 3: $k=1$ case: Left, the square of the wave packet $|\psi(u, v)|^{2}$ for $\chi=3.5$ and $N=15$. Right, the contour plot of the same figure with the classical path superimposed as the thick solid line.
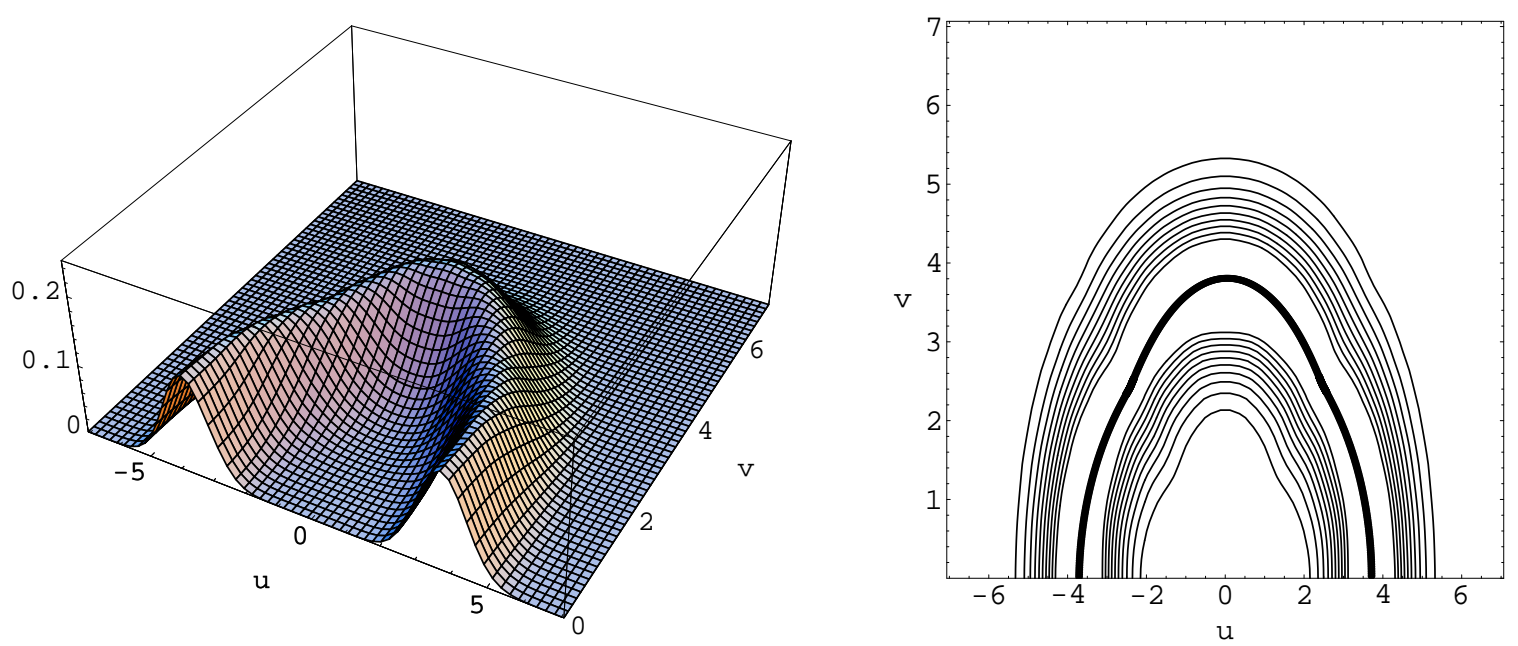

Figure 4: $k=-1$ case: Left, the square of the wave packet $|\psi(u, v)|^{2}$ for $\chi=3.5$ and $N=15$. Right, the contour plot of the same figure with the classical path superimposed as the thick solid line. 


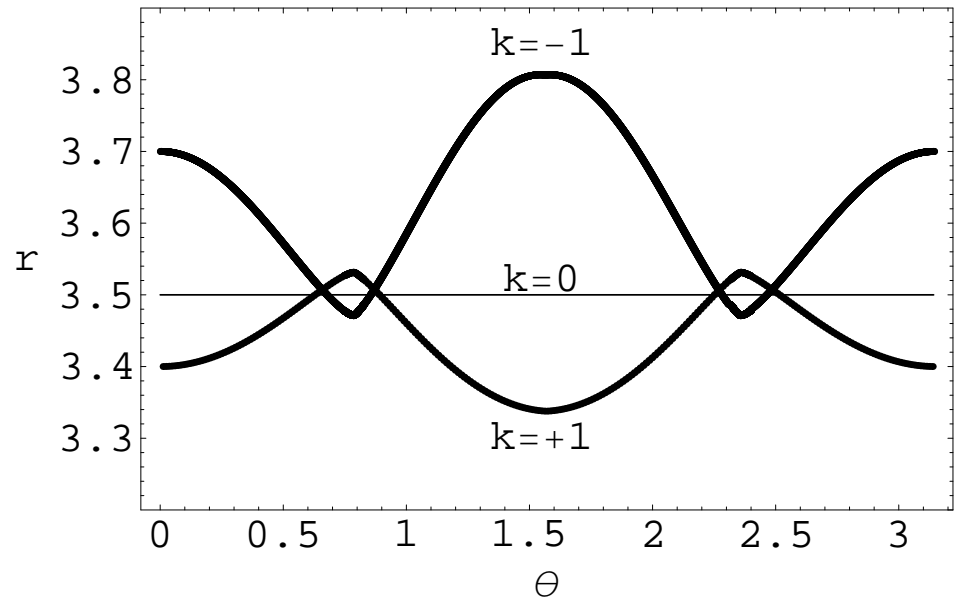

Figure 5: Classical paths for $k=\{1,0,-1\}$, where $r=\sqrt{u^{2}+v^{2}}$ and $\theta=\arctan (v / u)$. 